\title{
Exome sequencing reveals a novel TTC19 mutation in an autosomal recessive spinocerebellar ataxia patient
}

Hiroyuki Morino ${ }^{1 *}$, Ryosuke Miyamoto ${ }^{1}$, Shizuo Ohnishi ${ }^{2}$, Hirofumi Maruyama ${ }^{1}$ and Hideshi Kawakami ${ }^{1}$

\begin{abstract}
Background: Spinocerebellar ataxias (SCAs) are heterogeneous diseases characterized by progressive cerebellar ataxia associated with dysarthria, oculomotor abnormalities, and mental impairment. To identify the causative gene, we performed exome sequencing on a Japanese patient clinically diagnosed with recessive SCA.

Method: The patient is a 37-year-old Japanese woman with consanguineous parents. The head magnetic resonance imaging (MRI) showed cerebellar atrophy and T1 low/T2 high intensity at the bilateral inferior olives. Single-nucleotide polymorphism (SNP) genotyping and next-generation sequencing were performed, and the variants obtained were filtered and prioritized.

Results: After these manipulations, we identified a homozygous nonsense mutation of the TTC19 gene (p.Q277*). TTC19 has been reported to be a causative gene of a neurodegenerative disease in Italian and Portuguese families and to be involved in the pathogenesis of mitochondrial respiratory chain complex III (cIII) deficiency. This report is the first description of a TTC19 mutation in an Asian population. Clinical symptoms and neuroimaging are consistent with previous reports. The head MRI already showed abnormal features four years before her blood lactate and pyruvate levels were elevated.

Conclusions: We should consider the genetic analysis of TTC19 when we observe such characteristic MRI abnormalities. Genes associated with mitochondrial function cause many types of SCAs; the mutation we identified should help to elucidate the pathology of these disorders.
\end{abstract}

Keywords: Exome sequencing, Mitochondrial respiratory chain complex III, Nonsense mutation, Spinocerebellar ataxia, TTC19

\section{Background}

Spinocerebellar ataxias (SCAs) are genetically, clinically and pathologically heterogeneous diseases characterized by progressive cerebellar ataxia variably associated with dysarthria, oculomotor abnormalities, epilepsy, and mental impairment. Neuronal loss is observed in cerebellum and brainstem pathologies, and neuroimaging demonstrates the atrophy of those regions. These pathologies can be caused by autosomal dominant, autosomal recessive, and X-linked mutations, and many of the dominant

\footnotetext{
* Correspondence: morino@hiroshima-u.ac.jp

'Department of Epidemiology, Research Institute for Radiation Biology and Medicine, Hiroshima University, 1-2-3, Kasumi, Minami-ku, Hiroshima 734-8553, Japan

Full list of author information is available at the end of the article
}

mutations are caused by CAG triplet repeat expansion. Thirteen recessive SCAs (numbered as SCAR1-13) have been reported, and causative genes have been identified for 7 of them.

In recent years, it has been reported that cases of neurodegenerative disease associated with atrophy of the cerebellar vermis and the cerebral cortex are caused by homozygous nonsense mutations of TTC19 [GenBank: NM_017775] in Italian and Portuguese families [1,2]. TTC19 encodes tetratricopeptide repeat domain 19 which consists of 380 amino acids. TTC19 is involved in the assembly and activity of ubiquinol-cytochrome $c$ reductase (mitochondrial respiratory chain complex III, E.C.1.10.2.2) (MRC cIII). Mammalian cIII consists of 11 subunits [3]. Among them, cytochrome $b$ is the only 
gene encoded by mitochondrial DNA; all other subunits are encoded by nuclear DNA. Mutations in BCS1L [4], one of the assembly factors, are involved in most cases of cIII deficiency. According to the report mentioned above [1], the TTC19 protein is another assembly factor of cIII, and nonsense mutations in its gene cause the functional loss of cIII and, consequently, neurodegenerative disease.

Here, we performed exome sequencing on a patient clinically diagnosed with recessive SCA and identified a novel TTC19 mutation. Her main symptoms were cerebellar ataxia and mental impairment, and magnetic resonance imaging (MRI) results were similar to those described in previous reports $[1,2]$.

\section{Methods}

\section{Patient}

We performed exome sequencing on a Japanese woman born to consanguineous parents who were first cousins (Figure 1A). The 37-year-old woman noticed dysarthria at age 31. She exhibited brisk tendon reflex, ataxic gait, severe truncal ataxia, horizontal nystagmus, intellectual impairment, and pes cavus. Head MRI showed cerebellar atrophy and T1 low/T2 high intensity at the bilateral inferior olives at the age of 33 (Figure 2). Her blood lactic acid (14.0 mg/dl, normal range: $3-17 \mathrm{mg} / \mathrm{dl})$ and pyruvic acid levels (0.62 mg/dl, normal range: $0.30-0.94 \mathrm{mg} / \mathrm{dl})$ were normal at the age of 35 but were elevated at the age of 37 (lactic acid: $24.9 \mathrm{mg} / \mathrm{dl}$, pyruvic acid: $1.63 \mathrm{mg} / \mathrm{dl}$ ). A muscle biopsy was not performed because the patient declined consent. She began to use a wheelchair when she was 34 years old.
Identity by descent (IBD)

SNP genotyping was performed using the Genome-Wide Human SNP Array 6.0 (Affymetrix, Santa Clara, CA, USA). Identity by descent (IBD) was calculated using Homozygosity Mapping software [5].

\section{Exome sequencing}

Genomic DNA (gDNA) was extracted from the peripheral lymphocytes of the patients. gDNA libraries were prepared using the SeqCap EZ Human Exome Library v2.0 (Roche NimbleGen, Madison, WI, USA). Sequencing was performed with 100-bp paired-end reads on a HiSeq2000 sequencer (Illumina, San Diego, CA, USA). We used BWA (http://bio-bwa.sourceforge.net/) [6] for alignment and mapping, Samtools (http://samtools.sourceforge.net/) [7] and Picard (http://picard.sourceforge.net/) for SAM/ BAM handling, GATK (http://www.broadinstitute.org/ gatk/) [8] and Samtools for variant calls, and Annovar (http://www.openbioinformatics.org/annovar/) [9] for annotation. Functional predictions due to amino acid changes were estimated using PolyPhen2 (http://genetics. bwh.harvard.edu/pph2/) [10], SIFT (http://sift.bii.a-star.edu. sg/) [11], and Mutation Taster (http://www.mutationtaster. org/index.html) [12]. We evaluated copy number variants (CNVs) from exome reads using CoNIFER (http://conifer. sourceforge.net/) [13]. Control exome sequences were obtained from Japanese patients undergoing exome analysis for diseases other than SCAs. All reported genomic coordinates were in GRCh37/hg19. The identified mutations were validated with a standard polymerase chain reaction (PCR)based amplification followed by sequence analysis with an
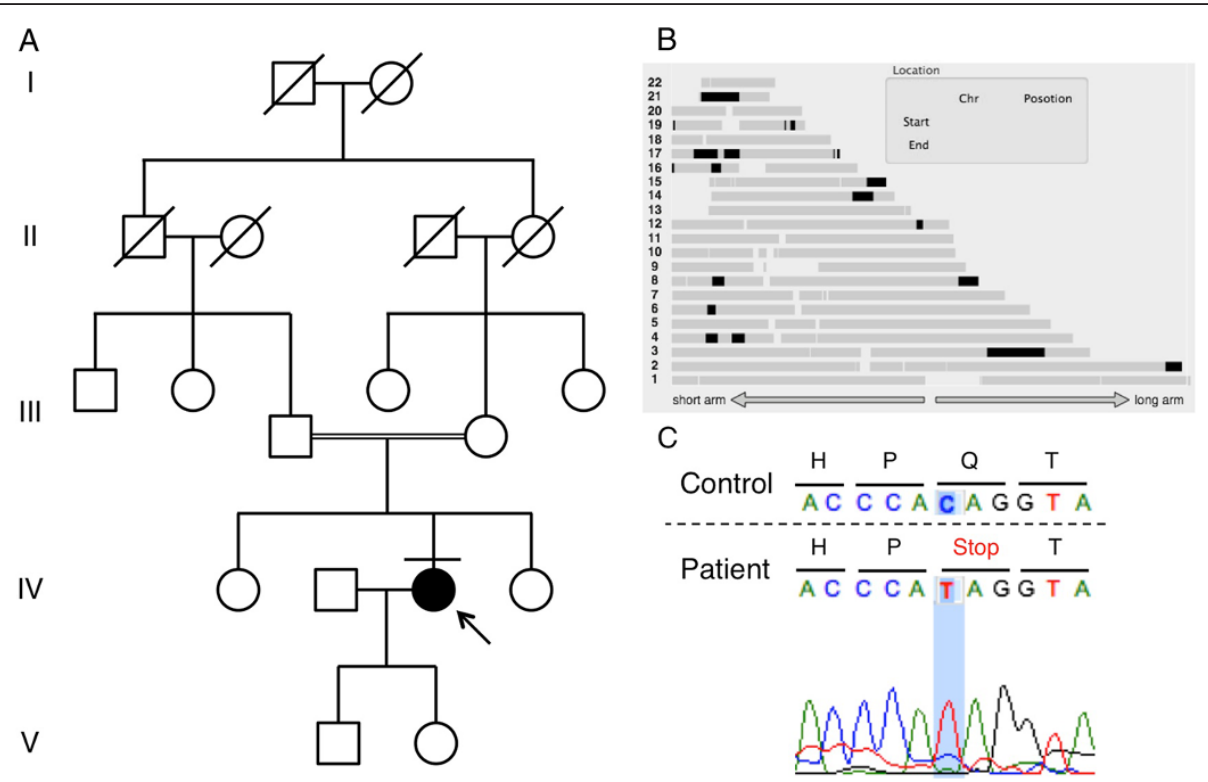

Figure 1 Pedigree chart, homozygosity mapping, and capillary sequencing. A. The patient was born to consanguineous parents who were first cousins. No other members of the family have exhibited the same symptoms. B. Black bar indicates IBD. Relatively long segments of IBD exist on chromosomes 3, 17, and 21. C. Patient has a homozygous nonsense mutation of TTC19 (c.829C > T, p.Q277*). 


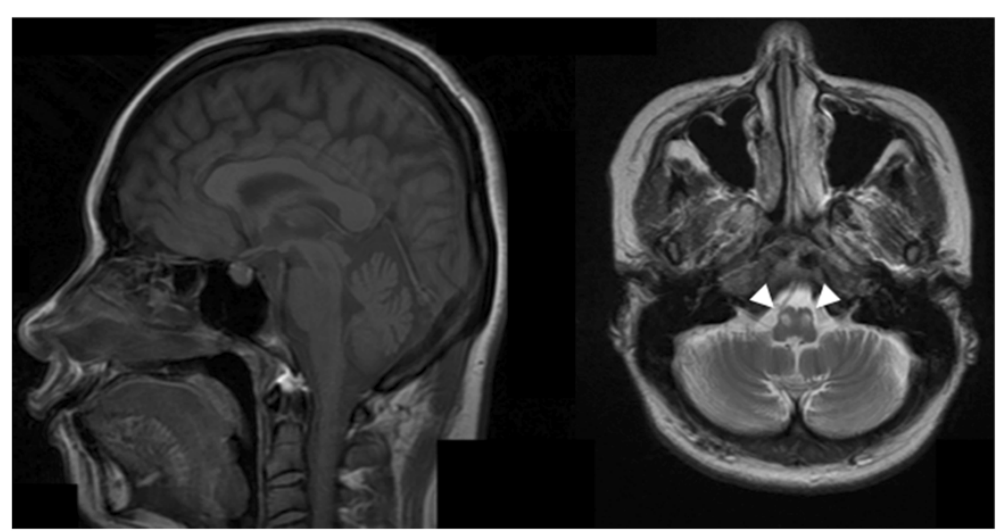

Figure 2 Brain MRI. Left is T1 weighted sagittal image, and right is T2 weighted axial image. Cerebellar atrophy was observed. Bilateral inferior olives showed T2 high intensity (arrowheads).

Applied Biosystems 3130 DNA sequencer (Life Technologies, Carlsbad, CA, USA). Additionally, we enrolled 180 normal Japanese controls without any neurological disorder and screened them for this mutation with the capillary sequencer. The research procedure was approved by the ethics committee of Hiroshima University. All examinations were performed after obtaining written informed consent from the patient and control cohort for publication of this case report and any accompanying images.

\section{Results}

\section{IBD}

The total length of IBD is $136,462,257 \mathrm{bp}$, and the percentage of IBD is $5.2 \%$. There are 1,283 genes in the IBD regions on the RefSeq database. ATXN3 and PRKCG, causative genes of SCA3 and SCA14 respectively, are located in the IBD regions, but there are no known associated genes with recessive SCAs. Relatively long segments of IBD exist on chromosomes 3, 17, and 21 (Figure 1B). IBD analysis was used to subsequently refine the candidates.

\section{Exome sequencing}

The results of the exome sequencing are shown in Table 1. There were 100,134,744 total reads and 90,942,725 mapped reads, and the mean coverage was 90 . We obtained 98,277 variants from GATK and Samtools. We used filtering criteria consisting of zygosity, genomic position, function, open databases (dbSNP build 135, 1000 genomes and ESP 5400), regions of IBD, and population-matched controls, which resulted in several sequence variants for each sample. There were no variant candidates in the known causative genes of SCDs. From the results of filtering, 4 candidate mutations were identified in the genes of NPM2, PDLIM2, TTC19 and ZSCAN4 (Additional file 1: Table S1). One of these mutations was a homozygous nonsense mutation of the TTC19 gene (GenBank: NM_017775:c.829C > T, p.Q277*), which was the most likely candidate to cause SCAs. The presence of this mutation was confirmed using capillary sequencing (Figure 1C). This TTC19 mutation was identified in a heterozygous state in one of the 145 population-matched disease control samples but not in the 180 normal control samples. The heterozygous sample was an odontopathy patient without any neurological deficit, and the $\mathrm{CNV}$ data indicated no deletions or duplications of the TTC19 gene.

\section{Discussion}

This report describes a TTC19 mutation causing ataxia and metal impairment in an Asian population. According to the previous reports, all patients harbored homozygous nonsense mutations in TTC19, and head MRI showed characteristic findings including cerebellar atrophy and abnormal intensity at the bilateral inferior olives $[1,2]$. Our patient had a novel homozygous nonsense mutation of TTC19, and head MRI were quite similar to those previously reported. The TTC19 protein is an assembly factor of MRC cIII, which transfers electrons from coenzyme Q to cytochrome c. TTC19 mutations

Table 1 Result of exome sequencing

\begin{tabular}{ll}
\hline Sequencing data & \\
\hline Total reads & $100,134,744$ \\
Mapped reads & $90,942,725$ \\
Mean coverage & 90 \\
Variant & \\
Total & 98,277 \\
Not found in public DBs & 8,699 \\
AA substitution & 873 \\
Homozygote & 76 \\
Not found in in-house DB & 20 \\
IBD & 4 \\
Nonsense & 1 \\
\hline
\end{tabular}


lead to mitochondrial dysfunction, which causes increased levels of blood lactic acid. Simultaneously, magnetic resonance spectroscopy shows a lactic peak [1]. While the patient's head MRI revealed abnormal features, her blood lactic acid and pyruvic acid levels were normal. However, these acid levels were elevated 6 years after the disease onset. One should consider mitochondrial abnormality and perform genetic analysis when observing such MRI characteristics. In the previous studies, no TTC19 protein was detected and the TTC19 transcript level was markedly reduced in samples with TTC19 mutations $[1,2]$. However, further analysis could not be performed because we could not obtain any additional clinical specimens from the patient other than gDNA.

We identified one heterozygote for this mutation in control samples. This result indicates an allele frequency of $0.14 \%$, but this mutation was not included in public SNP databases such as dbSNP and 1000 genomes. Therefore, the actual frequency of the mutation is even lower, which does not rule out the possibility that the mutation is a cause of recessive inheritance of the symptoms observed in the patient.

Neurodegeneration caused by mutations of TTC19 are classified as mitochondrial complex III deficiencies (MC3DNs), including MC3DN1 [MIM:124000] [4], which is associated with compound heterozygous or homozygous mutations of the BCS1L gene. Clinical symptoms of MC3DN are varied, but in reports on mutations of TTC19, many cases exhibit neurological disorders in adulthood, and some cases present both hemiplegia and cerebellar ataxia. Pyramidal signs were not observed in our case, but intellectual dysfunction was observed. As shown in previous reports, TTC19 p.Q173Rfs*4, p.L219* [1] and p.A200Afs*8 [2] are located between the first and the second TPR domains, but p.Q277*, the novel substitution we identified is located between the second and third domains, accordingly deleting half of the TPR domains. Notably, all these mutations are nonsense mutations. Clinical symptoms were mild compared with the symptoms from previously reported cases, but determining whether mutations are associated with clinical symptoms may require a longer observation of our patient's clinical course and the accumulation of more cases.

Regarding other variant candidates, the NPM2 variant was predicted as benign by PolyPhen-2, Mutation Taster, and SIFT; the ZSCAN4 variant was predicted as damaging only by SIFT. Thus, it is unlikely that NPM2 or ZSCAN4 is the causative mutation. The PDLIM2 variant was predicted as disease causing by Mutation Taster and SIFT. However, it is unlikely that PDLIM2 is the cause of SCA because PDLIM2 is expressed at low levels in the brain and is speculated to be associated with the inflammatory response of $\mathrm{T}$ helper 17 cells $[14,15]$.
Several studies have implicated mutations in genes involved in mitochondrial function as a cause of SCAs [16]. For example, coenzyme Q10 deficiencies are known as diseases that lead to ataxia associated with MRC dysfunction. Thus far, there have been 6 subtypes of coenzyme Q10 deficiencies, including COQ10D4 [MIM:612016], which is also referred to as SCAR9 and is caused by homozygous or compound heterozygous mutations in $C A B C 1$ [17]. In addition to cerebellar ataxia, coenzyme Q10 deficiencies show hypotonia, epilepsy, and muscular symptoms. Thus, mutations in the genes causing mitochondrial dysfunction show broad spectrum of clinical outcome.

\section{Conclusions}

In conclusion, using exome sequencing, we identified a new TTC19 mutation that is the cause of an autosomal recessive SCA. Notably, our case showed abnormal MRI findings before we detected a metabolic disorder. Genes associated with mitochondrial function cause many types of SCAs, and we should consider the genetic analysis of mitochondria-related genes when observing such characteristic clinical features including MRI abnormalities.

\section{Additional file}

Additional file 1: Table S1. Candidate SNPS after variant filtering.

\section{Competing interests}

Dr. Morino, Dr. Miyamoto, Dr. Ohnishi, Dr. Maruyama and Dr. Kawakami report no conflicts of interest.

\section{Authors' contributions}

HMo contributed substantially to conception and design, the acquisition of data, the analysis and interpretation of data, and the drafting of the article. RM participated in the acquisition of data, as well as the analysis and interpretation of the data. SO participated in the acquisition of samples and clinical data. HMa substantially contributed to conception and design, drafting the article, and the acquisition of funding. HK substantially contributed to conception and design, the acquisition of data, the acquisition of funding, and general supervision of the research group. All authors critically revised the article for important intellectual contents, and approved the final manuscript.

\section{Acknowledgements}

This work was supported in part by the Funding Program for Next Generation World-Leading Researchers from the Cabinet Office of the Government of Japan (HMa), a Grant-in-Aid for Scientific Research on Innovative Areas (Brain Environment) from the Ministry of Education, Science, Sports and Culture of Japan (HK), and a Grant-in-Aid from the Research Committee of CNS Degenerative Diseases from the Ministry of Health, Labour and Welfare of Japan (HK).

\section{Author details}

${ }^{1}$ Department of Epidemiology, Research Institute for Radiation Biology and Medicine, Hiroshima University, 1-2-3, Kasumi, Minami-ku, Hiroshima

734-8553, Japan. ²Department of Neurology, Tanabe Neurological Hospital, Fujiidera, Osaka, Japan.

Received: 6 September 2013 Accepted: 2 January 2014

Published: 7 January 2014 


\section{References}

1. Ghezzi D, Arzuffi P, Zordan M, Da Re C, Lamperti C, Benna C, D'Adamo P, Diodato D, Costa R, Mariotti C, Uziel G, Smiderle C, Zeviani M: Mutations in TTC19 cause mitochondrial complex III deficiency and neurological impairment in humans and flies. Nat Genet 2011, 43:259-263.

2. Nogueira C, Barros J, Sá MJ, Azevedo L, Taipa R, Torraco A, Meschini MC Verrigni D, Nesti C, Rizza T, Teixeira J, Carrozzo R, Pires MM, Vilarinho L, Santorelli FM: Novel $T T C 19$ mutation in a family with severe psychiatric manifestations and complex III deficiency. Neurogenetics 2013, 14:153-160.

3. Iwata S, Lee JW, Okada K, Lee JK, Iwata M, Rasmussen B, Link TA, Ramaswamy S, Jap BK: Complete structure of the 11-subunit bovine mitochondrial cytochrome $b c_{1}$ complex. Science 1998, 281:64-71.

4. Blázquez A, Gil-Borlado MC, Morán M, Verdú A, Cazorla-Calleja MR, Martín MA, Arenas J, Ugalde C: Infantile mitochondrial encephalomyopathy with unusual phenotype caused by a novel $B C S 1 L$ mutation in an isolated complex III-deficient patient. Neuromuscul Disord 2009, 19:143-146.

5. Hagiwara K, Morino H, Shiihara J, Tanaka T, Miyazawa H, Suzuki T, Kohda M, Okazaki Y, Seyama K, Kawakami H: Homozygosity mapping on homozygosity haplotype analysis to detect recessive disease-causing genes from a small number of unrelated, outbred patients. PLoS One 2011, 6:e25059.

6. Li H, Durbin R: Fast and accurate short read alignment with Burrows-Wheeler transform. Bioinformatics 2009, 25:1754-1760.

7. Li H, Handsaker B, Wysoker A, Fennell T, Ruan J, Homer N, Marth G, Abecasis G, Durbin R, 1000 Genome Project Data Processing Subgroup: The Sequence Alignment/Map format and SAMtools. Bioinformatics 2009, 25:2078-2079.

8. DePristo MA, Banks E, Poplin R, Garimella KV, Maquire JR, Hartl C, Philippakis AA, del Angel G, Rivas MA, Hanna M, McKenna A, Fennell TJ, Kernytsky AM, Sivachenko AY, Cibulskis K, Gabriel SB, Altshuler D, Daly MJ: A framework for variation discovery and genotyping using next-generation DNA sequencing data. Nat Genet 2011, 43:491-498.

9. Wang K, Li M, Hakonarson H: ANNOVAR: functional annotation of genetic variants from high-throughput sequencing data. Nucleic Acids Res 2010, 38:e164.

10. Ramensky V, Bork P, Sunyaev S: Human non-synonymous SNPs: server and survey. Nucleic Acids Res 2002, 30:3894-3900.

11. Ng PC, Henikoff S: Predicting deleterious amino acid substitutions. Genome Res 2001, 11:863-874.

12. Schwarz JM, Rödelsperger C, Schuelke M, Seelow D: MutationTaster evaluates disease-causing potential of sequence alterations. Nat Methods 2010, 7:575-576.

13. Krumm N, Sudmant PH, Ko A, O'Roak BJ, Malig M, Coe BP, NHLBI Exome Sequencing Project, Quinlan AR, Nickerson DA, Eichler EE: Copy number variation detection and genotyping from exome sequence data. Genome Res 2012, 22:1525-1532.

14. Loughran G, Healy NC, Kiely PA, Huigsloot M, Kedersha NL, O'Connor R: Mystique is a new insulin-like growth factor-I-regulated PDZ-LIM domain protein that promotes cell attachment and migration and suppresses Anchorage-independent growth. Mol Biol Cell 2005, 16:1811-1822.

15. Tanaka T, Yamamoto Y, Muromoto R, Ikeda O, Sekine Y, Grusby MJ, Kaisho T, Matsuda T: PDLIM2 inhibits T helper 17 cell development and granulomatous inflammation through degradation of STAT3. Sci Signal 2011, 4:85

16. Zeviani M, Simonati A, Bindoff LA: Ataxia in mitochondrial disorders. Handb Clin Neurol 2012, 103:359-372.

17. Mollet J, Delahodde A, Serre V, Chretien D, Schlemmer D, Lombes A, Boddaert N, Desguerre I, de Lonlay P, de Baulny HO, Munnich A, Rötig A: $C A B C 1$ gene mutations cause ubiquinone deficiency with cerebellar ataxia and seizures. Am J Hum Genet 2008, 82:623-630.

doi:10.1186/1471-2377-14-5

Cite this article as: Morino et al:: Exome sequencing reveals a novel TTC19 mutation in an autosomal recessive spinocerebellar ataxia patient. BMC Neurology 2014 14:5.

\section{Submit your next manuscript to BioMed Central and take full advantage of:}

- Convenient online submission

- Thorough peer review

- No space constraints or color figure charges

- Immediate publication on acceptance

- Inclusion in PubMed, CAS, Scopus and Google Scholar

- Research which is freely available for redistribution

Submit your manuscript at www.biomedcentral.com/submit
Biomed Central 